\title{
Use and effectiveness of oral analgesia when fitting an intrauterine device
}

Judy Murty, MB ChB, MFFP, SCMO, Contraception and Sexual Health Services, Leeds South Primary Care NHS Trust, Burmantofts Health Centre, Leeds, UK

Correspondence: Dr J Murty, Contraception and Sexual Health Services, Leeds South Primary Care NHS Trust, Burmantofts Health Centre, Cromwell Mount, Leeds LS9 7ST, UK. E-mail: murty@easynet.co.uk

(Accepted 3rd January 2003)

Journal of Family Planning and Reproductive Health Care 2003; 29(3): 150-151

\begin{abstract}
Objective. To explore (a) if analgesia taken before an intrauterine device (IUD) fitting actually lessens the discomfort of the fitting and (b) whether there is a relationship between anxiety and choosing to take pain relief before an IUD fitting.

Population. Women having an IUD fitted in participating clinics in Leeds between 1 April and 31 July 2001.

Method. Advice was given to women who were having an IUD fitted to take analgesia before they came to the clinic if they wished. The women completed a questionnaire at the time of fitting to record anticipated discomfort, actual discomfort and the use of analgesia before the fitting. A second questionnaire was sent to women 6 months later.

Response. A total of 109 women completed the first questionnaire and 54 completed the second questionnaire. Findings and discussion. Women who were more anxious and who anticipated that the procedure would be painful, even if it was not as bad as expected at the time of the fitting, will recall that it was a painful experience. Those who chose to take painkillers before the procedure may identify this group of women. Higher anxiety levels may contribute to the perceived discomfort.
\end{abstract}

\section{Introduction}

The discomfort that a woman may feel when having an intrauterine device (IUD) fitted can vary from slight discomfort to severe cramping. In the Leeds service, advice was given to women who were having an IUD fitted to take analgesia before they came to the clinic if they wished. The women pre-booking an IUD fitting were given the information in a leaflet together with other general advice, such as continuing their current method of contraception until the IUD fitting.

Over the years that IUDs have been fitted there has been little research conducted into the relief of the discomfort of fitting. The cervical nerve innervation is different to that of the body of the uterus, ${ }^{1}$ and studies have generally looked at cervical analgesia and the discomfort of using a tenaculum. ${ }^{2,3}$ Comments seem to suggest that IUD fitting is not as painful as expected. ${ }^{2,4}$ There is little evidence about the effect of analgesics on the uterine cramping during an IUD fitting. Evidence from other gynaecological conditions shows that ibuprofen is the most effective analgesia for uterine cramping. ${ }^{5}$ Stress and emotional response can also affect the pain gateways and affect the perception of the discomfort of a gynaecological procedure. 6

One of the questions in the service was whether analgesia taken before an IUD fitting actually lessened the discomfort of the fitting. If it did, then was there a need to offer analgesia to all women before a fitting?

\section{Method}

Women having an IUD fitted between 1 April and 31 July 2001 were asked to complete a questionnaire at the time of the fitting and were asked if they could be contacted
6 months later with a second questionnaire. Ethical approval was obtained from the Local Research Ethics Committee (LREC).

The first questionnaire asked the women their opinion on the service they obtained and whether they had sufficient information regarding the IUD fitting. As part of the questionnaire the women were asked to complete a visual analogue score (VAS) of their anticipated discomfort, the discomfort at the time and whether they had any analgesia before the fitting. The VAS was graded from 1 for very little discomfort to 10 for severe pain. The follow-up questionnaire asked the women to record their recollection of the score at the fitting on the same VAS.

The IUDs were fitted at general clinics and specialist IUD training clinics. The doctors and nurses were asked to follow their normal procedures when fitting the IUD. The service uses Instillagel for cervical analgesia and it was recorded if used.

The data were entered into an SPSS statistical package (SPSS, Chicago, IL, USA) and the variables were compared using various tests including the Chi-squared test, Wilcoxon signed rank test and Mann-Whitney $U$-test. The results were considered significant if $\mathrm{p}<0.05$

\section{Results}

First questionnaire

A total of 214 devices were fitted in the defined time period but not all of the clinics participated in the completion of questionnaires. One hundred and nine women completed the questionnaire at the time of fitting. Ninety-two women indicated that we could contact them again. The age distribution at the time of fitting was bimodal, with a peak in the group aged 20-25 years and a second peak in the age group 30-34 years. Twenty-seven women were nulliparous, and 78 had a full-term pregnancy.

A total of 42 Nova T380 and 49 Mirena were fitted. The other IUDs fitted included Gyne-T380, Multiload and GyneFix. Instillagel was used at the time of fitting for 69

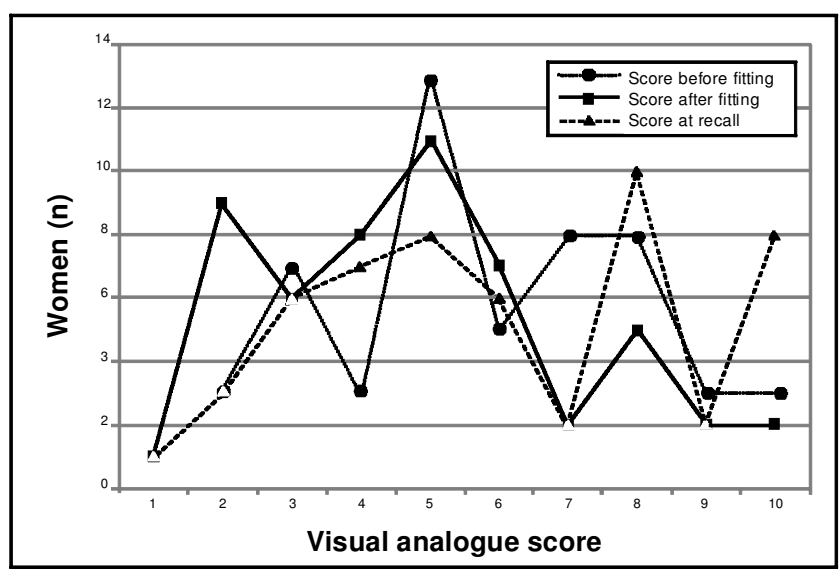

Figure 1 Visual analogue score of the 54 women who replied to the second questionnaire 
women; 30 women had taken analgesia before fitting. Of those women who had taken analgesia, ibuprofen was the most commonly used drug (11 women).

The scores as recorded on the visual analogue line showed significance in the pain scores before and after fitting with the pain score at fitting less than anticipated (Wilcoxon signed rank test, $\mathrm{p}=0.0008$ ). Those women who had taken analgesia before fitting expected a higher pain score (Mann-Whitney $U$-test, $\mathrm{p}=0.0046$ ) but the score of this group at fitting was not significantly different from the rest of the population. The nulliparous woman tended to expect a higher pain score, while the parous woman tended to have a lower score after fitting. These differences were not, however, significant with the Mann-Whitney $U$-test. There was no statistical difference in the scores depending on whether Instillagel was used or not.

\section{Second questionnaire}

Fifty-four women $(57 \%$ of those who indicated we could contact them) returned the second questionnaire. This population was different from the original sample as they were more likely to have taken analgesia before the fitting ( $p$ $=0.01)$. The recollected score after 6 months for the fitting was considerably higher than that recorded immediately after the fitting (Wilcoxon signed rank test, $\mathrm{p}=0.003$ ) (Figure 1).

\section{Discussion}

From these results it appears there is a group of women who are more anxious and anticipate that the procedure will be painful, even if it was not as bad as expected at the time of fitting, and will recall that it was a painful experience. This group of women may be identified by those who have taken painkillers before the procedure. Higher anxiety levels can contribute to the perceived discomfort being worse than it actually is. The group that responded to the second questionnaire could have been more proactive and involved in the IUD fitting than the main population and therefore more willing to reply to the second questionnaire.

The discomfort of the IUD fitting is not only related to the use of analgesia but also to the anxiety of the woman undergoing the procedure. Those women anticipating a painful procedure become more anxious and were probably more likely to take analgesia before the fitting. Even though the reported discomfort at fitting is no different from the main population, this particular group of women still recalls the experience as being worse than it really was. Oral analgesia is no longer routinely offered to women having an IUD fitted in our service. Greater emphasis is placed on reducing anxiety before and during a fitting. If oral analgesia is requested then ibuprofen is advised.

Statements on funding and competing interests

Funding. None identified.

Competing interests. None identified.

\section{References}

1 Goldstuck ND. The management of intrauterine device related pain. Br J Fam Plann 1979; 5: 63-65.

2 Seamark C. Is the fitting of an intrauterine contraceptive device a painful experience? Br J Fam Plann 1993; 19: 227-229.

3 Oloto EJ, Bromham DR, Murty JA. Pain and discomfort perception at IUD insertion - effect of short-duration, low volume, intracervical application of two percent lignocaine gel (Instillagel) - a preliminary study. Br J Fam Plann 1996; 22: 177-180.

4 Hollingworth B. Pain control during insertion of an intrauterine device. Br J Fam Plann 1995; 21: 102-103.

5 Analgesics for primary dysmenorrhoea. Bandolier. 17 June 2002. www.jr2.ox.ac.uk/bandolier/booth/painpag/Chronrev/Analges/CP06 6.htm

6 Conference round up. London: new light on chronic pelvic pain in women. Trends Urol Gynaecol Sex Health 2002; 7(2): 11-14.

\section{But is she positive?}

\section{T T}

Your next patient is pregnant but doesn't want to be.

Where next? As a registered charity since 1968, bpas has offered affordable abortion care for women. We provide almost 50,000 abortions a year (including service agreements) and can offer all the professional help your patient needs.

bpas has a nationwide network of clinics and consultation centres. There are no long waits for appointments. We can offer a choice of times, clinics and procedures. All it takes to arrange an appointment is one call to the bpas Actionline on 08457304030.

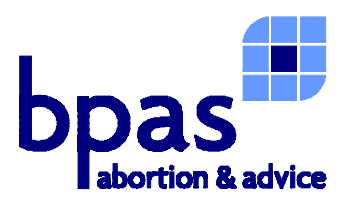

ACTIONLINE 08457304030

\section{bpas positively the best service}

